\title{
No impact without data access
}

\section{A considerable proportion of the usefulness and interest of research publications in our field comes from the data and associated metadata. We therefore insist that data be available for peer reviewers to see and readers to use. Authors should use public permanent repositories designed for appropriately consented data.}

A commonly expressed opinion is that there is value in restricting access to research data, where "ownership of data represents intangible capital that gives scientists a competitive advantage in some academic fields" (Nature $520,587,2015)$ - unless the proud owners of the data wish to publish in this journal that is, as readers expect there to be substance behind the claims of the research article and to be able to use those data in their own research. Funding bodies in many countries insist upon data access no later than their first publication as a condition for grants. There are now many options for secure and permanent data archiving in accordance with the wishes of the research subjects who gave informed consent for their data to be used for research.

The journal continues to support and endorse two major international databases. On page 692, Ilkka Lappalainen and colleagues present the operation and uses of the European Genome-phenome Archive (EGA), a secure repository for consented human genetics data. Another attractive option, the database of Genotype and Phenotype (dbGaP), was described here last year (Nat. Genet. 46, 934-938, 2014). We note that these databases offer two models for access. In the EGA, data access committees for each institution, country or funder work with data users, and the repository provides infrastructure and access control accordingly. dbGaP, in contrast, is centralized at the US National Institutes of Health, and the Institutes provide access committees for the data from work they fund.

Although we recognize that these US and European databases are suitable for most research in the field, national laws may require local databases and access protocols to be developed for different communities. The most positive benefit that could be accrued by local data stewardship would be capacity building through using data access to recruit qualified international experts to collaborate or work locally on the data. But, given the global reach of the internet and cloud, capacity could be built electronically as well as in person, so we urge forward-looking strategists and legislators to anticipate these benefits rather than to be unnecessarily restrictive.

We regard a data descriptor and a live accession code to a permanent data set in a supported repository as the minimum acceptable data access provision compatible with publication in a high-impact journal, and therefore hold the view that restrictive legislation with regard to access to data will inevitably place local researchers at an international disadvantage with respect to reputation, publication and collaboration. Without specific access provisions for qualified applicants to use data for purposes for which they were originally consented, such costive data management will also undermine trust in the research.

As we are an international journal and usable repositories have been developed and supported by funders and researchers alike, we see no reason to make exceptions or concessions to review or publish research articles-from any part of the world-that lack the most basic access to data. In practice, we will contact the authors of articles where much of the impact of the work depends on access to the data and ask either that the data resources be properly described and deposited or that the authors obtain a data descriptor with Scientific Data (http://www.nature.com/sdata/) to explain their data access provisions. In addition to guiding users to the data, this process helps with deposition in a suitable repository in a maximally useful format. A high-impact paper needs to have a high degree of usefulness to others. 